\title{
The Gerbode Defect: About 2 Cases
}

\author{
Amine Majdoub, Anas Elhafidi, Cedric Mutuale, Salaheddine Boulmakoul, Mohammed Messouak
}

Faculty of Medicine and Pharmacy of Fez, Department of Cardiovascular Surgery, Sidi Mohammed Ben Abdellah University, CHU HASSAN II FEZ, Fez, Morocco

Email: aminemajdoub82@gmail.com

How to cite this paper: Majdoub, A., Elhafidi, A., Mutuale, C., Boulmakoul, S. and Messouak, M. (2020) The Gerbode Defect: About 2 Cases. World Journal of Cardiovascular Surgery, 10, 115-121. https://doi.org/10.4236/wjcs.2020.107014

Received: May 11, 2020

Accepted: July 7, 2020

Published: July 10, 2020

Copyright $\odot 2020$ by author(s) and Scientific Research Publishing Inc. This work is licensed under the Creative Commons Attribution International License (CC BY 4.0).

http://creativecommons.org/licenses/by/4.0/

\begin{abstract}
Background: Left ventricular to right atrial communications (LV-RA) or the Gerbode defects are rare and complex types of ventricular septal defect. Their clinical diagnosis is not specific. However, the main clue to identifying Gerbode defect comes from Doppler-coupled echocardiography (TTE), and the treatment is mainly surgical. Aim: We hereby report our experience in surgical management of Gerbode defect through two exceptional congenital cases (type 1 and type 2 of Gerbode defect). Case Presentation: The diagnosis was established in adult patients, the first case is a 27-year-old woman who had dyspnea, and in whom the TTE revealed LV-RA communication, with repercussions on the right cavities. The second case is a 23-year-old man, with the concept of statutory weight delay, consulted for progressive dyspnea; the TTE initially revealed a very large perimembranous ventricular defect associated with significant pulmonary hypertension. Cardiopulmonary bypass surgery was done for a successful and complete correction. Conclusion: Gerbode defect is so rare; the diagnosis is made by TTE. And surgery must not be delayed until repercussion on right cavities and pulmonary hypertension.
\end{abstract}

\section{Keywords}

Congenital Disease, Ventricular Septal Defect, Cardiopulmonary Bypass

\section{Introduction}

Left ventricular to right atrial communication (LV-RA) or the Gerbode defectis a rare type of cardiac malformations estimated at less than one percent of all congenital heart diseases [1]. It is a complex and varied set of membrane septum abnormalities. We report two cases, one direct (the first case) and one indirect (the second case), that have been fully corrected under Cardiopulmonary bypass surgery.

\section{Observations}

1st Case: A 27-year-old woman without specific-history has consulted for stage 
III NYHA dyspnea with progressive evolution to stage III associated with palpitations, effortless fatigability without chest pain. The cardiovascular examination finds a systolic breath radiating in wheel radius with a maximum intensity at the 3rd intercostal space in left parasternal. Her ECG follows a regular sinus rhythm with a deflected axis to the right. A TTE (Figure 1) showed a $7-10 \mathrm{~mm}$ VSD shunting between the LV and RA with a maximum gradient of $120 \mathrm{mmHg}$; with as a resounding dilation of the RA, RV and Thickened tricuspid valves, tricuspid ring dilated at $40 \mathrm{~mm}$, also a severe tricuspid regurgitation by diastasis. The inter-auricular septum is flat. No vegetation or thrombus is visible.

The surgical procedure under cardiopulmonary bypass consisted, after right atriotomy, in the closure of a large defect of $10 \mathrm{~mm}$ diameter located above the Todaro tendon opposite the septal leaflet of the tricuspid valve (Figure 2), by four separated pledged sutures (with Ti-cron 4-0). The important tricuspid leak

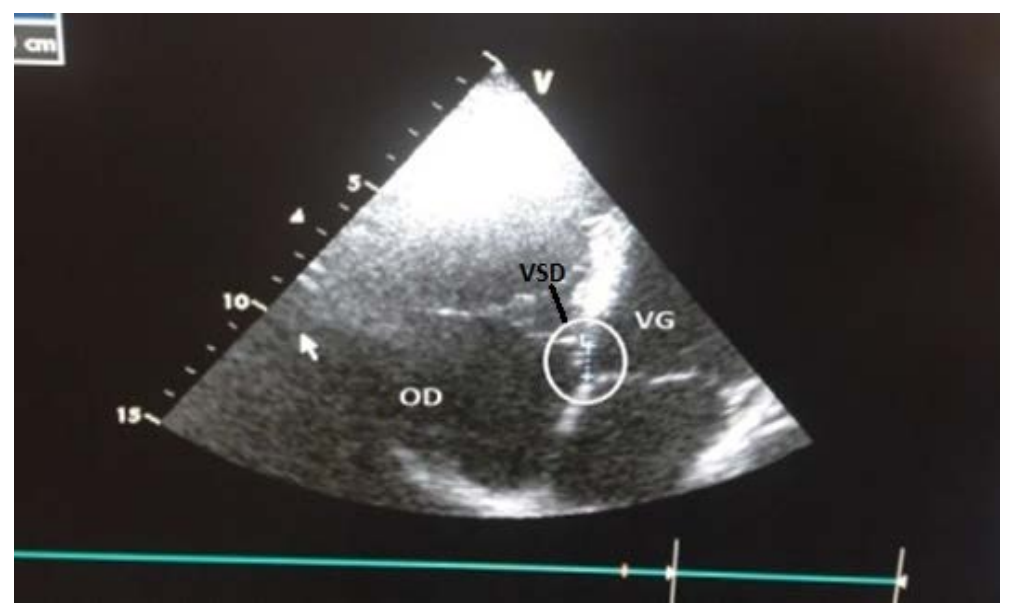

Figure 1. Echocardiographic image (1st case): 7 - $10 \mathrm{~mm}$ VSD shunting between LV and RA. Abbreviations: $\mathrm{OD}=$ right atrium, $\mathrm{VG}=$ left ventricle, $\mathrm{VSD}=$ ventricle septal defect.

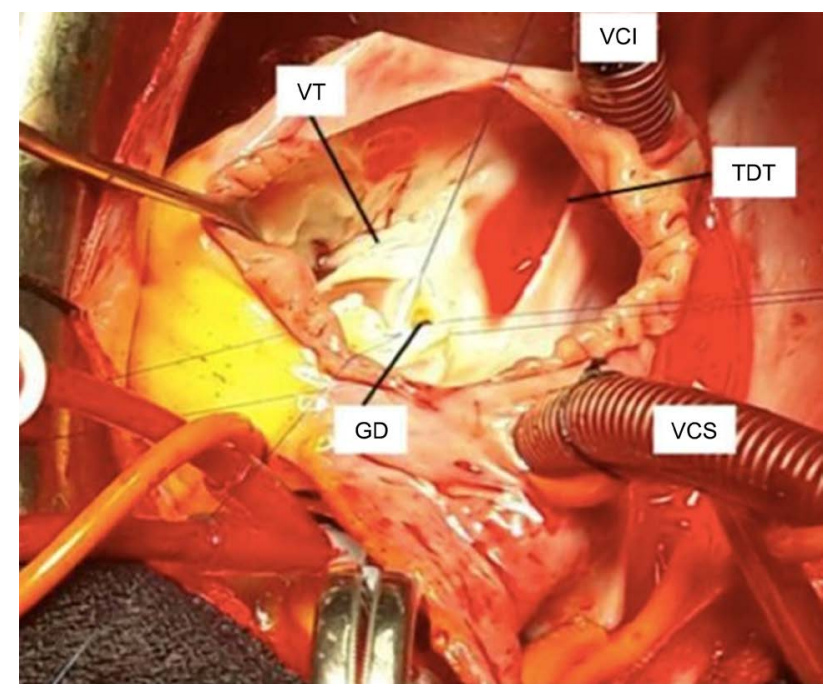

Figure 2. Operative view through right atriotomy: LV-RA direct shunt (type 2). Abbreviations: $\mathrm{GD}=$ Gerbode defect type $2, \mathrm{TDT}=$ Todaro, $\mathrm{VT}=$ tricuspid valve, $\mathrm{VCI}=$ inferior vena cava, VCS = superior vena cava. 
was corrected by a 3D ring Number 32. The aortic clamping time was $43 \mathrm{~min}$ and cardiopulmonary bypass time was $1 \mathrm{~h} 7 \mathrm{~min}$.

The postoperative follow-up was marked by a transient arrhythmia atrial fibrillation controlled by amiodarone. The postoperative TTE confirmed the absence of residual shunt and the correction of tricuspid leakage to a minimum. The patient was discharged on Day 8. Another TTE control was performed a year later and returned in favor of a significant regression in the size of the right cavities, no residual shunt.

2nd Case: A 23-year-old man with a notion of weight-loss delay (weight: $32 \mathrm{~kg}$, height: $1 \mathrm{~m} \mathrm{45}$ ) has consulted for a progressive dyspnea evolving since childhood associated with palpitations and cyanosis on exercise. The cardiovascular examination finds a systolic breath radiating in wheel radius and the pulmonary B2 is intensively increasing. His ECG follows a regular sinus rhythm with a deflected axis to the right, the thorax X-Ray objective a Cardiomegaly (cardiothoracic index at 0.69 ) and hyper pulmonary vascularization.

An ETT initially revealed a very wide per-membranous ventricular defect $(\sim 20$ $\mathrm{mm}$ ) partially closed by the tricuspid valve, at the color Doppler: shunt left-right between the left ventricle and the right ventricle with a maximum gradient of 93 $\mathrm{mmHg}$, associated with moderate tricuspid regurgitation, significant pulmonary hypertension (120 $\mathrm{mmHg})$, dilatation of the left cavities and RA.

During the procedure under cardiopulmonary bypass, and after right atriotomy, we found a defect in tricuspid valve tissue, and after the suspension of the septal leaflet we discovered a large defect of about $20 \mathrm{mmHg}$ shunting of the LV to RA communication through the septal leaflet, then was clogged by a PTFE patch using ten 5.0 pledged Prolene sutures passed in U. On the tricuspid valve, we realized a reconstruction of the septal and posterior sheets and then an annuloplasty type semi DeVega was performed for tricuspid leakage correction. The aortic clamping time was $1 \mathrm{~h} 7 \mathrm{~min}$ and the cardiopulmonary bypass time was 1 h 26 min (see Figure 3).
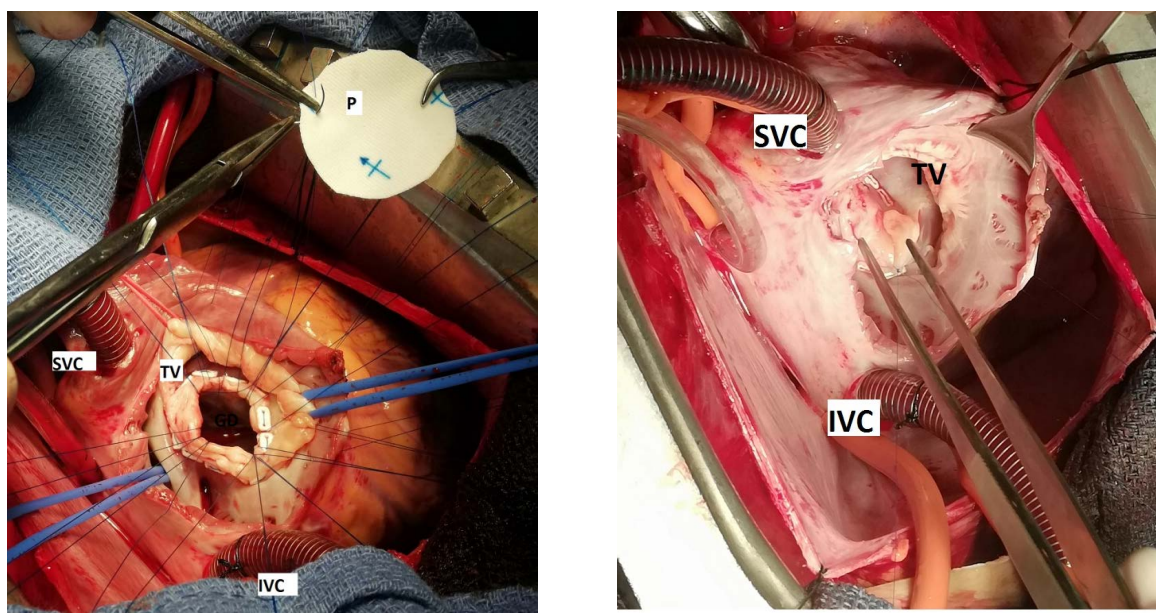

Figure 3. (Before and after repair). Operative view through right atriotomy: LV-RA indirect shunt (type 1). SVC: superior vena cava, IVC: inferior vena cava, TV: tricuspid valve, P: Patch, GD: Gerbode defect type 1. 
The postoperative follow-up (day 4) was marked by a transient atrioventricular block without hemodynamic repercussions which spontaneously regressed. The postoperative TTE confirmed the absence of residual shunt and the correction of the tricuspid leak. The patient was discharged on day 9. Another TTE control was performed a year later returned in favor of a significant regression in the size of the left cavities, no pulmonary hypertension $(30 \mathrm{mmHg})$ and no residual shunt.

\section{Discussion}

Left ventricular to right atrial (LV-RA) communications was first described in the 19th century by BUHL [2]. It is however to GERBODE that one owes the first series of surgical correction in 1957 in the USA [3]. The Gerbode defect is a rare form of shunt from the LV to RA due to either congenital or acquired causes, and estimated less than one percent of all congenital heart diseases [1].

Understanding Gerbode shunt means understanding the membranous septum and the structures it separates. This septum is divided into two portions based on the implantation of the septal leaflet of the tricuspid valve: the apical portion is interventricular while the basal portion is atrioventricular. Since the implantation of the tricuspid valve is one centimeter below that of the mitral valve, it is easy to imagine that the atrioventricular portion separates the RA and the hunting chamber from the LV [4] (see Figure 4).

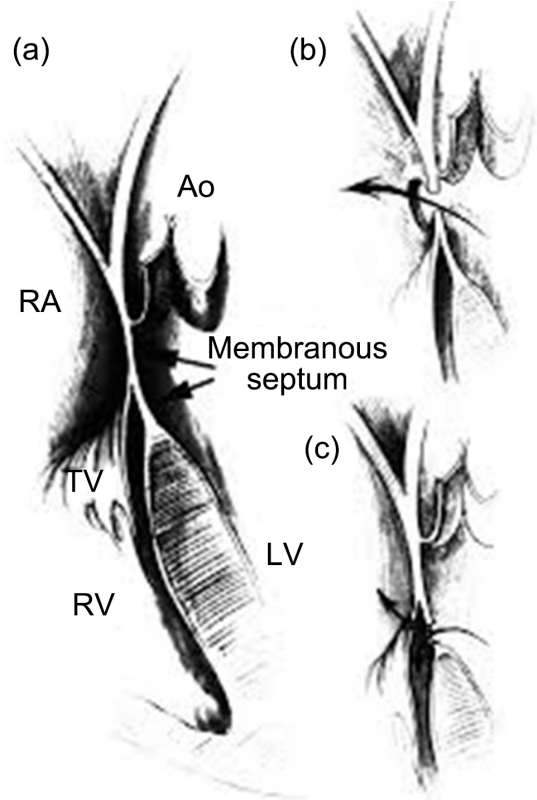

Figure 4. Anatomic relationship of the septal leaflet of the tricuspid valve to the membranous septum [4]. (a) The septal leaflet divides the membranous septum into interventricular and atrioventricular portions; (b) A supravalvular defect within the atrioventricular septum permits shunting directly from the left ventricle to the right atrium; (c) An infravalvular defect within the interventricular septum is associated with a perforation of the septal leaflet of the tricuspid valve. Abbreviations: $\mathrm{RA}=$ right atrium; $\mathrm{RV}=$ right ventricle, Ao $=$ aorta. 
The defect occurs in the membranous portion of the interventricular septum above the level of the tricuspid valve. Thus, two types of Gerbode shunts are distinguished:

- The indirect type (type 1) which is the most common. The interventricular apical portion that carries a defect and generally associated with a jet lesion on the septal tricuspid leaflet that it can perforate, thus creating an indirect shunt in the RA.

- The direct type (type 2) where it is the basal atrial-ventricular portion which has a gap causing a reflux in the RA [1].

These septal anomalies can be whether acquired often or congenital as shown in Table 1. However, the clinical features are the same as ventricular septal defect.

The diagnosis is made often by TTE, thereby cardiac catherisation is exceptional. The natural history of the disease is cardiac cavities dilatation and pulmonary hypertension. The best option for treatment is surgery under cardiopulmonary bypass [5] [6].

We report here two rare types of Gerbode defect. They are among the oldest in the literature, which explains the symptoms of heart failure and annihilates the illusion of spontaneous closure. The first, which is a restrictive VG-OD shunt, corresponds to type 2. In the second case, the interventricular defect passes through the septal leaflet of the tricuspid and corresponds to type 1. Although the notion of consanguinity has not been found, the etiology of the shunt, in both cases, is of congenital origin, late discovery. This explains the symptoms of heart failure, as well as the repercussions reported in both cases on the cardiac cavities and the degree of pulmonary arterial hypertension (PAH).

If in the first case, one TTE was sufficient to establish the diagnosis. On the other hand for the second case, the diagnosis was made intraoperatively. Thus before any abnormal dilation of the RA and a strong maximum gradient, the Gerbode shunt must be suspected.

Whatever the type of shunt, the right atriotomy approach allows a complete correction of the cardiopathy as well as the tricuspid regurgitation caused by the jet lesion on the septal leaflet or by hyper-flow.

Table 1. Causes of Gerbode shunt [7].

\section{Causes of Gerbode shunt}

1) Congenital

2) Acquired++
a) Post-surgical: mitral, aortic, tricuspid, atrial septal defect (Primum and/or Secundum)
b) Infectious endocarditis
c) Myocardial infarction of the right coronary
d) Blunt cardiac trauma 
The majority of teams used a PATCH to plug the breach; our experience of 4 0 separated points seemed satisfactory for the small defect. The late age of the intervention also explains the importance of the tricuspid leak corrected by a 3D ring, in the first case, and in the other, by the DeVega technique.

A transient atrioventricular block in the second case (predictable) [3] and a classical atrial arrhythmia as postoperative rhythm disorder in second case has marked the postoperative follow-up.

Our surgical approach with the right atriotomy allowed us to correct both the septal defect and the tricuspid leak, with an excellent long-term result: a total absence of a residual shunt in both cases with two different techniques (direct closure by separate points or patch), thus, a perfect seal of the tricuspid valve whether it is repaired with a $3 \mathrm{D}$ ring or with a DeVega.

Complete surgical repair has allowed our patients to recover a clear improvement and a disappearance of symptoms in the long term, also proven by TTE control: normal sized cavities, absence of shunt, tricuspid insufficiency and residual PAH.

\section{Conclusion}

The Gerbode shunt is part of the complex and varied set of septal defect of the membranous septum, whose proximity to the conduction bundles and the anatomical relationships require a complete but prudent surgical management. Positive diagnosis by an experienced sonographer or pediatric cardio seems easy. The late age of the operation is, however, associated with more or less significant repercussions on the right heart chambers.

\section{Consent}

Informed consent was obtained from our patients whose cases are described.

\section{Conflicts of Interest}

The authors declare no conflicts of interest regarding the publication of this paper.

\section{References}

[1] Kelle, A.M., Young, L., Kaushal, S., Duffy, C.E., Anderson, R.H. and Backer, C.L. (2009) The Gerbode Defect: The Significance of a Left Ventricular to Right Atrial Shunt. Cardiology in the Young, 19, 96-99. https://doi.org/10.1017/S1047951109991685

[2] Meyer, H. (1857) Uber angeborene Enge oder Verschluss der Lungenarterienbahn. Archiv für pathologische Anatomie und Physiologie und für klinische Medicin, 12, 497-538. https://doi.org/10.1007/BF01950079

[3] Gerbode, F., et al. (1958) Syndrome of Left Ventricular-Right Atrial Shunt. Successful Repair of Defect in Five Cases, with Observation of Bradycardia on Closure. Annals of Surgery, 148, 433-446. https://doi.org/10.1097/00000658-195809000-00012

[4] Riemenschneider, T.A. and Moss, A.J. (1967) Left Ventricular-Right Atrial Com- 
munication. The American Journal of Cardiology, 19, 710-718.

https://doi.org/10.1016/0002-9149(67)90476-6

[5] Tidake, A., Gangurde, P. and Mahajan, A. (2015) Gerbode Defect-A Rare Defect of Atrioventricular Septum and Tricuspid Valve. Journal of Clinical and Diagnostic Research, 9, OD6-OD08. https://doi.org/10.7860/JCDR/2015/14259.6531

[6] Jeffrey, J., Kamran, M., Handwerker, S., Kumar, N. and Marcali, M. (2009) The Gerbode Defect: Left Ventricular to Right Atrial Communication-Anatomic, Hemodynamic, and Echocardiographic Features. Echocardiography, 26, 993-998. https://doi.org/10.1111/j.1540-8175.2009.01009.x

[7] Carpenter, R.J. et al. (2012) Gerbode Defect Associated with S. lugdunensis Native Valve Infective Endocarditis Requiring Cardiac Surgery. Journal of Cardiac Surgery, 27, 316-320. https://doi.org/10.1111/j.1540-8191.2011.01379.x 